\title{
Dual wavelength multiple-angle light scattering system for cryptosporidium detection
}

\author{
S. Buaprathoom ${ }^{\mathrm{a}^{*}}$, S. Pedley ${ }^{\mathrm{b}}$, and S. J. Sweeney ${ }^{\mathrm{a}}$ \\ ${ }^{a}$ Advanced Technology Institute, Faculty of Engineering and Physical Science, University of Surrey, \\ Surrey GU2 7XH, UK \\ ${ }^{\mathrm{b}}$ Postgraduate Medical School, Faculty of Health and Medical Sciences, University of Surrey, Surrey \\ GU2 7XH, UK
}

\begin{abstract}
A simple, dual wavelength, multiple-angle, light scattering system has been developed for detecting cryptosporidium suspended in water. Cryptosporidium is a coccidial protozoan parasite causing cryptosporidiosis; a diarrheal disease of varying severity. The parasite is transmitted by ingestion of contaminated water, particularly drinking-water, but also accidental ingestion of bathing-water, including swimming pools. It is therefore important to be able to detect these parasites quickly, so that remedial action can be taken to reduce the risk of infection. The proposed system combines multiple-angle scattering detection of a single and two wavelengths, to collect relative wavelength angle-resolved scattering phase functions from tested suspension, and multivariate data analysis techniques to obtain characterizing information of samples under investigation. The system was designed to be simple, portable and inexpensive. It employs two diode lasers (violet InGaN-based and red AlGaInP-based) as light sources and silicon photodiodes as detectors and optical components, all of which are readily available. The measured scattering patterns using the dual wavelength system showed that the relative wavelength angle-resolved scattering pattern of cryptosporidium oocysts was significantly different from other particles (e.g. polystyrene latex sphere, E.coli). The single wavelength set up was applied for cryptosporidium oocysts'size and relative refractive index measurement and differential measurement of the concentration of cryptosporidium oocysts suspended in water and mixed polystyrene latex sphere suspension. The measurement results showed good agreement with the control reference values. These results indicate that the proposed method could potentially be applied to online detection in a water quality control system.
\end{abstract}

Keywords: cryptosporidium detection, particle concentration, light scattering, particle characterization

\section{INTRODUCTION}

Contamination of water with pathogenic microorganisms are reported to be responsible for between two and five million deaths per year many of them children under the age of five. In developed countries, advanced water treatment plants remove much of the contamination, yet outbreaks can still occur due to the limitations of the present methods for detecting pathogens in water. Cryptosporidium is recognized as one of the principle contributors to water contamination. It is a coccidial protozoan parasite and the causative agent of an unpleasant illness called cryptosporidiosis. The symptom of cryptosporidiosis are diarrhea, mild abdominal pain, nausea and vomiting, mild fever and fatigue. The cryptosporidiosis may be very serious in people whose immune system is weakened or less effective such as the very young, the elderly, AIDS sufferers and those on immune-suppressant drugs. No drug has been shown to be effective against. Cryptosporidium infection and recovery from the illness is dependent on the body's immune system. The infection is transmitted by tiny spore-or egg-like cells call oocysts.

*s.buaprathoom@surrey.ac.uk; phone +44 (0)1483689409

Biophotonics: Photonic Solutions for Better Health Care III, edited by Jürgen Popp,

Wolfgang Drexler, Valery V. Tuchin, Dennis L. Matthews, Proc. of SPIE Vol. 8427, 84272K

(C) 2012 SPIE $\cdot$ CCC code: $1605-7422 / 12 / \$ 18 \cdot$ doi: $10.1117 / 12.921661$

Proc. of SPIE Vol. $842784272 \mathrm{~K}-1$ 
These oocysts are small, roughly spherical in shape and about 4 to $6 \mu \mathrm{m}$ in diameter ${ }^{1-4}$. The oocysts are transmitted to the patients by ingestion of contaminated water, particularly drinking-water, but also by accidental ingestion of bathingwater, including swimming pools ${ }^{5-6}$. It is therefore important to be able to detect these parasites quickly, so that remedial action can be taken to reduce the risk of infection.

A variety of techniques have been developed to detect cryptosporidium oocysts, including the polymerase chain reaction (PCR) ${ }^{7-8}$, reverse transcription PCR ${ }^{9}$, fluorescent in situ hybridization (FISH) ${ }^{10}$, tissue culture infectivity ${ }^{11}$ and immunofluorescence techniques ${ }^{12-13}$. These methods are time-consuming and require an experienced microscopist to identify the organisms.

Light scattering techniques are widely used to investigate the particle properties such as such as size ${ }^{14-16}$, refractive index ${ }^{17-18}$ and concentration ${ }^{19-20}$ and also applied to biological particle detection ${ }^{21-26}$ as its advantages; easy to perform; low maintenance; non-invasive; have high sensitivity; and the potential for real-time detection.

In this research, a multiple-angle light scattering system was developed for cryptosporidium detection. The proposed system was contributed by using single and dual wavelengths multiple-angle light scattering techniques. The single wavelength multiple-angle light scattering technique was applied for size and relative refractive index measurement and concentration measurement of cryptosporidium suspension in both monodispersion and mixed polystyrene latex sphere suspension. The dual wavelength multiple angle light scattering system was used to measure the relative wavelength scattering pattern from suspension as a means of particle selection. The system was designed to be simple, portable and inexpensive using low power diode lasers as light sources, Si photodiodes for detection and optical components, all of which are readily available.

\section{EXPERIMENTAL METHOD}

\subsection{Experimental set up and operation}

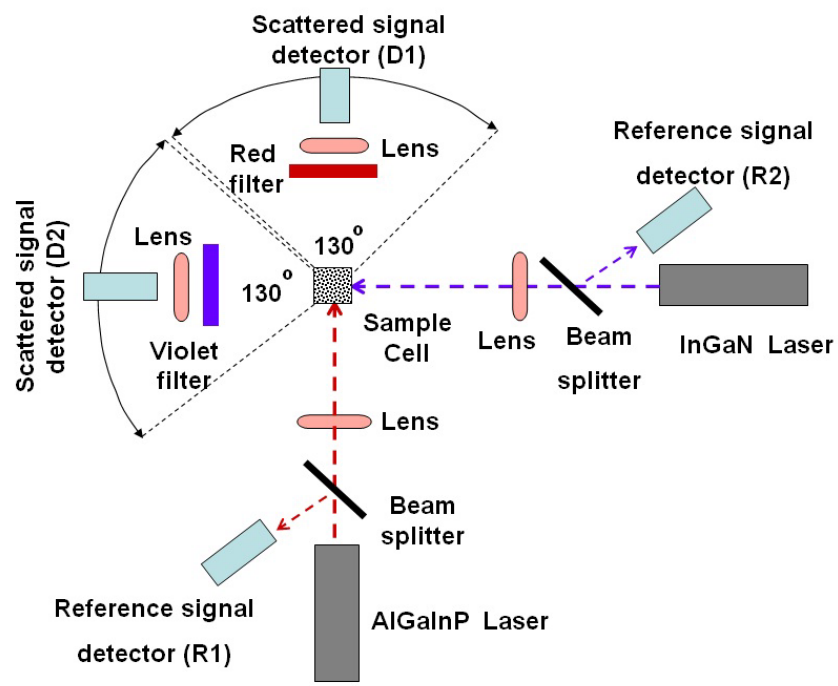

Figure 1 Schematic drawing of experimental set up.

The design of the multiple-angle light scattering system is shown in Figure 1. Two diode lasers (violet InGaN-based and red AlGaInP-based) were used as the light sources. The power to the diodes and the temperature of the diodes was controlled using source-meters (KEITHLEY 2420) and temperature controllers (LDT-5910B). The diodes were set to emit $5 \mathrm{~mW}$ perpendicular polarized light at 504.8 and $658.4 \mathrm{~nm}$ for violet and red lasers, respectively (measured by an 
optical power meter (ANRITSU ML910B), and an optical spectrum analyzer (Ando AQ 6315A)). Beam splitters (glass slides) were used to divide the light beams into reference signals detected by reference detectors $(\mathrm{R} 1, \mathrm{R} 2)$ for stabilizing the laser power and incident signals. Thin lenses $(\mathrm{f}=75 \mathrm{~mm})$ focused and directed the incident light beams onto the centre of a sample cell (glass cuvette with a cross-section of $10 \mathrm{~mm} \times 10 \mathrm{~mm}$ and a height of $50 \mathrm{~mm}$ ) containing sample suspension. Objective lenses (20x, 0.40NA) focused the scattered light signals onto the signal detectors (D1, D2). Red and violet filters were used as bandpass filters for scattered signals to detectors. Silicon photodiodes (AEPX 65) with buffer circuits were used as detecting systems for both the scattered and reference signals. The scattered signal detecting systems were mounted on a rotating base to collect the scattered signals through an angle from 5 to 65 in steps of one degree. All the operation was controlled via Labview ${ }^{\mathrm{TM}}$ software. The apparatus were also protected from external electromagnetic interference (which affected the performance of the detectors), airborne particles, surrounding light, and air-flow (which caused temperature fluctuation of the lasers) by covering with a metal box.

The set up was applied to measure the size and relative refractive index of cryptosporidium oocyst and concentration of the cryptosporidium suspension and by using single wavelength multiple angle light scattering technique (red laser was only operated). Multivariate data analysis technique was used to compose predictive models from data generated from Mie theory with corrections for size and relative refractive index measurement and calibration samples for concentration measurement. For the dual wavelength multiple-angle light scattering system was used to measure the relative wavelength scattering pattern as a means of particle selection.

\subsection{Multivariate data analysis}

The multivariate data analysis technique used in this experiment was Partial Least Square Regression (PLS-R) method. PLS-R is particularly useful when we need to predict a set of dependent variables or determination values $\left(\mathrm{Y}_{1}\right.$, $\left.\mathrm{Y}_{2}, \mathrm{Y}_{3}, \ldots\right)$ from a set of independent variables or measurement values $\left(\mathrm{X}_{1}, \mathrm{X}_{2}, \mathrm{X}_{3}, \ldots\right)$. It was used when typically the measurement of $\mathrm{Y}$ may be expensive, difficult, dangerous, labour intensive, etc. Its technique is to construct a multivariate regression model or predictive model using the information from standard data or calibration data to find the Y-relevant structure in $\mathrm{X}$ by successively extracting latent factors from both $\mathrm{X}$ and $\mathrm{Y}$ such that correlation between the extracted factors is maximized. The model then subsequently is used on new X measurements to predict new Y-values ${ }^{27}$. The schematic of the concept of the multivariate data analysis is shown in figure 2 .
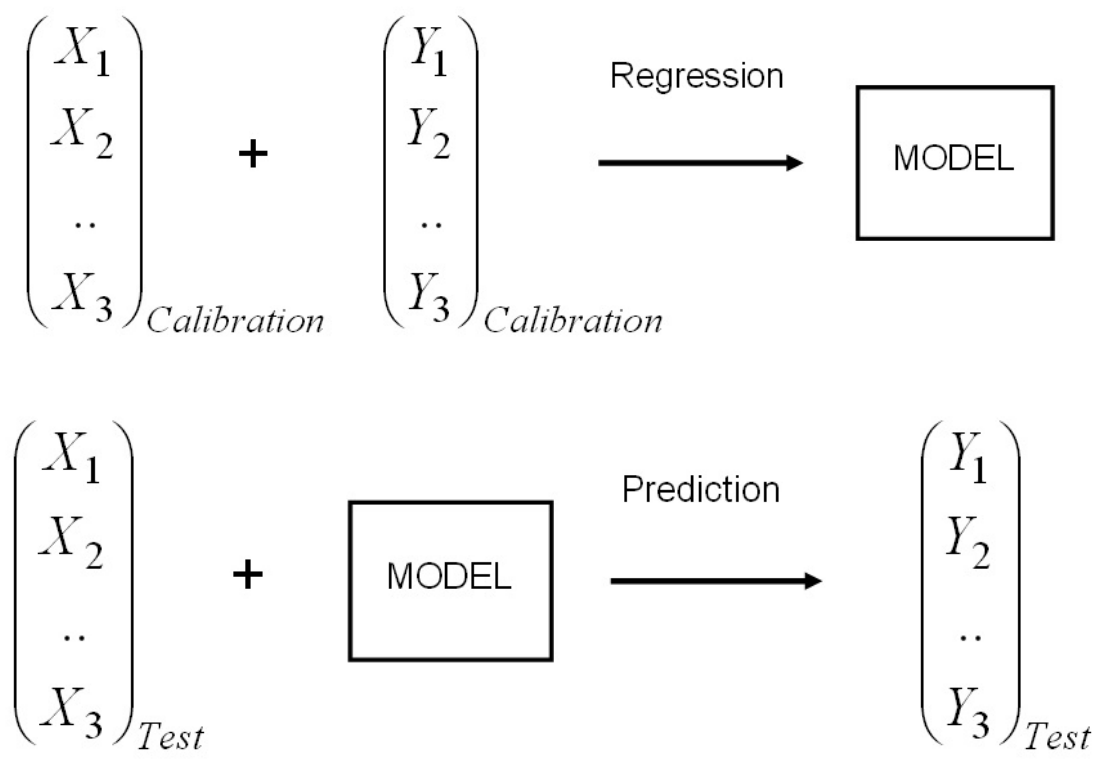

Figure 2 (a) Establishing a multivariate regression model from known $\mathrm{X}$ and $\mathrm{Y}$ data. (b) Using the multivariate regression model to predict new $\mathrm{Y}$ values from measurement variables $\mathrm{X}$ based on generated model. 


\section{CRYPTOSPORIDIUM MEASUREMENTS}

\subsection{Cryptosporidium's size and refractive index measurement}

As the scattered light intensity depended on the scattering particle's properties and the size and refractive index of the particle are fundamental physical and optical properties used to classify microorganism ${ }^{24}$, the set up system was applied to identify cryptosporidium oocyst by measuring their size and relative refractive index. The multiple angle light scattering system with single red wavelength described in section 2.1 was used to collect normalized angular resolved light scattering intensities from cryptosporidium oocysts suspended in water. In order to avoid the multiple scattering effects in the suspensions which would be impotence if the suspension is in high concentration, this experiment we used cryptosporidium oocysts suspension of $10^{5}$ oocysts/ml in deionized water (Supplied by Creative Science Company, UK). The transmittance coefficient of this sample was 0.976 which suggests that the multiple light scattering is not significant ${ }^{28}$. To interpret the scattered signal to get information from the sample, simulated angle dependent light scattering intensities from spherical particles by Mie theory ${ }^{29}$ were generated from a program called MiePlot 4.0 to form calibration data. As in the experiment the sample suspension was contained in the cuvette, therefore a correction by Snell's law and Fresnel refraction at the cuvette's faces was taken in account to the simulated scattered data. The corrected result of Mie theory is shown in the figure 3.

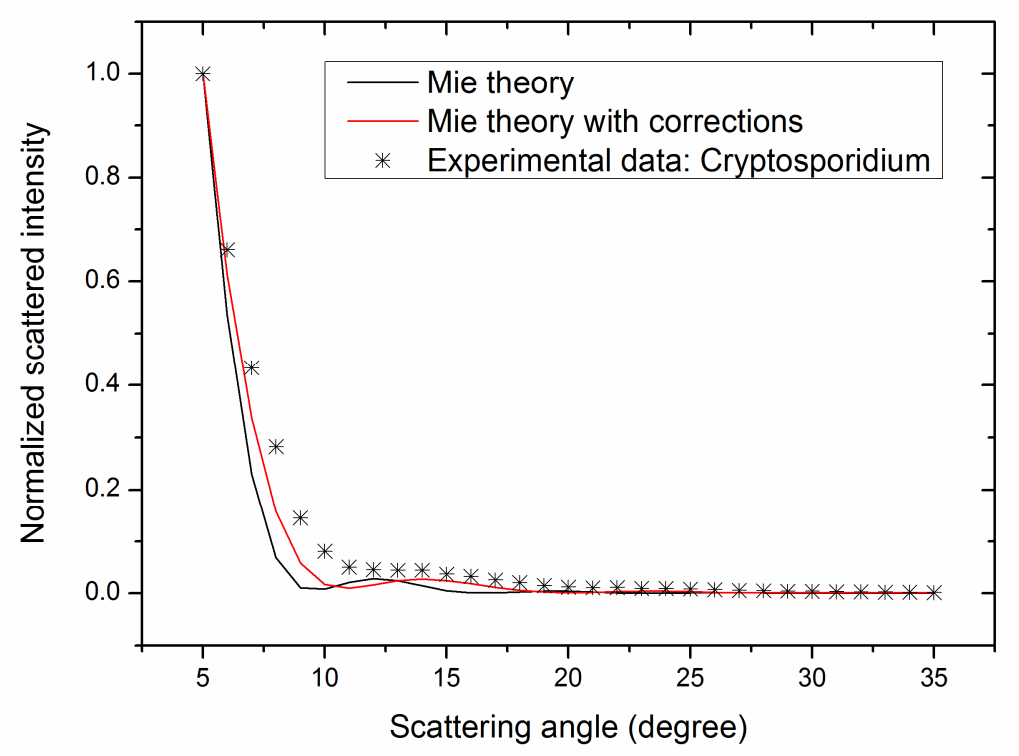

Figure 3. Scattered light from cryptosporidium oocysts, simulated scattered light from Mie theory and Mie theory with corrections.

In this experiment, the calibration data were generated from particle sizes from $0.1 \mu \mathrm{m}$ to $7 \mu \mathrm{m}$ in step $0.05 \mu \mathrm{m}$ and refractive index from 1.30-1.70 step 0.05. The PLS-R method was used to generate a model from the calibration data and predict the size and relative refractive index of the cryptosporidium oocysts in suspension based on the generated model. The predicted results from the scattered signal from the cryptosporidium oocysts suspended in the water are shown in figure 4. The predicted size of the cryptosporidium oocyst was $4.63 \pm 0.32 \mu \mathrm{m}$ which corresponds to the size of cryporsporidium oocysts measured from a Scanning Electron Microscope (SEM) as shown in figure $5(4.38 \pm 0.12 \mu \mathrm{m})$. The relative refractive index of the cryptosporidium predicted from the experiment was $1.398 \pm 0.081$ which consistent with the reference value of $1.369^{30}$. The measurement results proved that the presented method has potential to identify the cryptosporidium oocysts suspended in the water based on their size and refractive index. 

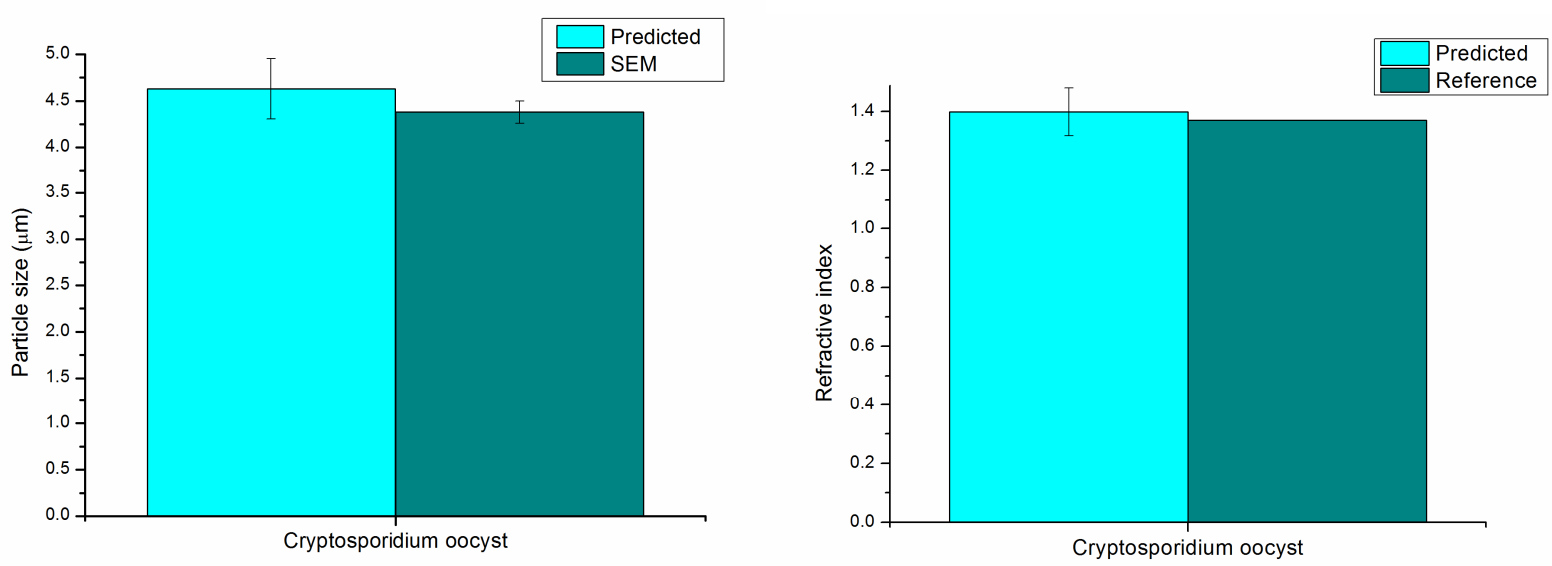

Figure 4 Measurement results of size and refractive index of cryptosporidium oocyst.

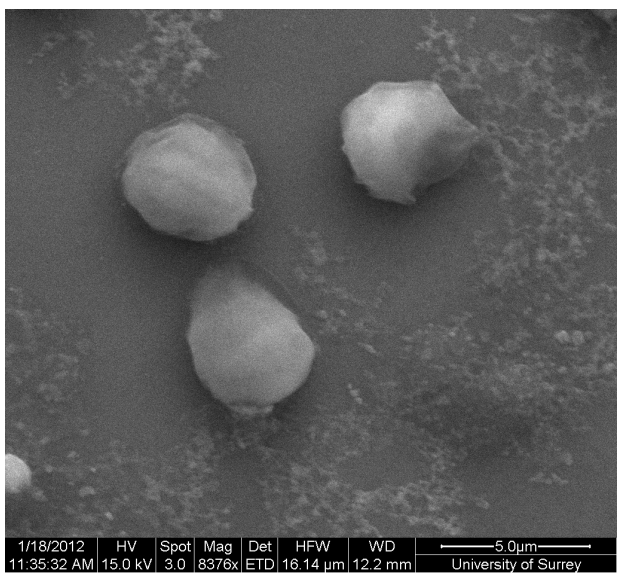

Figure 5 Scanning electron microscope image of the cryptosporidium oocyst.

\subsection{Cryptosporidium concentration measurement}

Beside of particle size and refractive index, light scattering intensity from suspension is also determined by the concentration of particles in the suspension medium. For suspension in low concentration (single scattering), the scattered light intensity can be considered from the addition of scattered light intensity by each particle. In monodispersed suspension, the scattered light intensity is linearly related to concentration, but not for poly-dispersed or mixed suspension in which cryptosporidium oocysts are usually found. By using the advantage of the combination of the multiple angle light scattering technique which consider scattered light from multiple scattering angles and the PLS-R method which is the statistical method for predicting the dependent variables from independent factors, the proposed system is able to measure differential of particle in both mono-dispersed and poly-dispersed suspension. In this experiment, the set up with single wavelength was applied to measure the concentration of cryptosporidium oocysts suspended in water in monodispersion and in mixed polystyrene suspension.

The concentration measurement system operates by collecting the angle dependent scattered light intensity from the calibration samples with known concentrations by using the presented multiple angle light scattering system with red light. The collected data of calibration samples are then used to generate a calibration or predictive model using the PLS$\mathrm{R}$ method. The scattered signals from investigated samples which are collected by the system are predicted based on the generated model to obtain the sample concentration. 


\section{Monodispersion}

The experimental samples of the monodispersed system were cryptosporidium oocyst suspended in de-ionized water. The predictive model was generated from cryptosporidium oocyst suspensions with concentration in the range of $10^{4}-10^{6}$ oocysts $/ \mathrm{ml}$. The angle dependent scattered light intensity of the calibration samples are presented in figure 6 . These scattered data were used to generate a predictive model by the PLS-R method. The regression results showed that the correlation between reference concentrations and predicted concentrations of the calibration samples was 0.989 . This meant the predictive model was reasonable to use to predict the concentration of test samples. The test samples (CT1-5) which prepared with different concentration from the calibration samples were measured the scattered angle dependent scattered signals as shown in figure 7a. The scattered signals from the test samples were predicted based on the composed predictive model. The predicted results are presented in figure $7 \mathrm{~b}$.

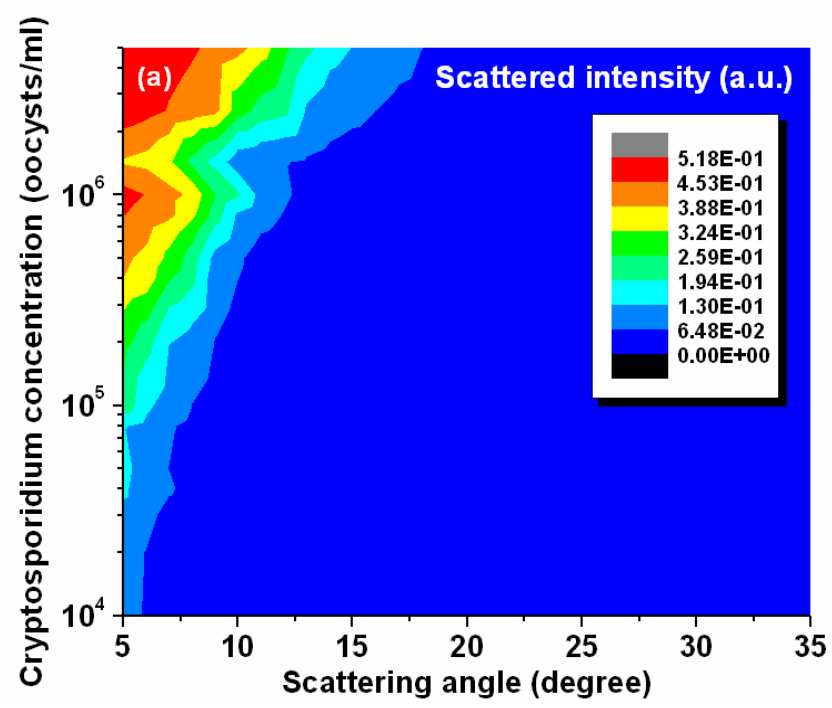

Figure 6. Angle dependent scattered intensities of calibration samples for cryptosporidium in mono-dispersed suspensions
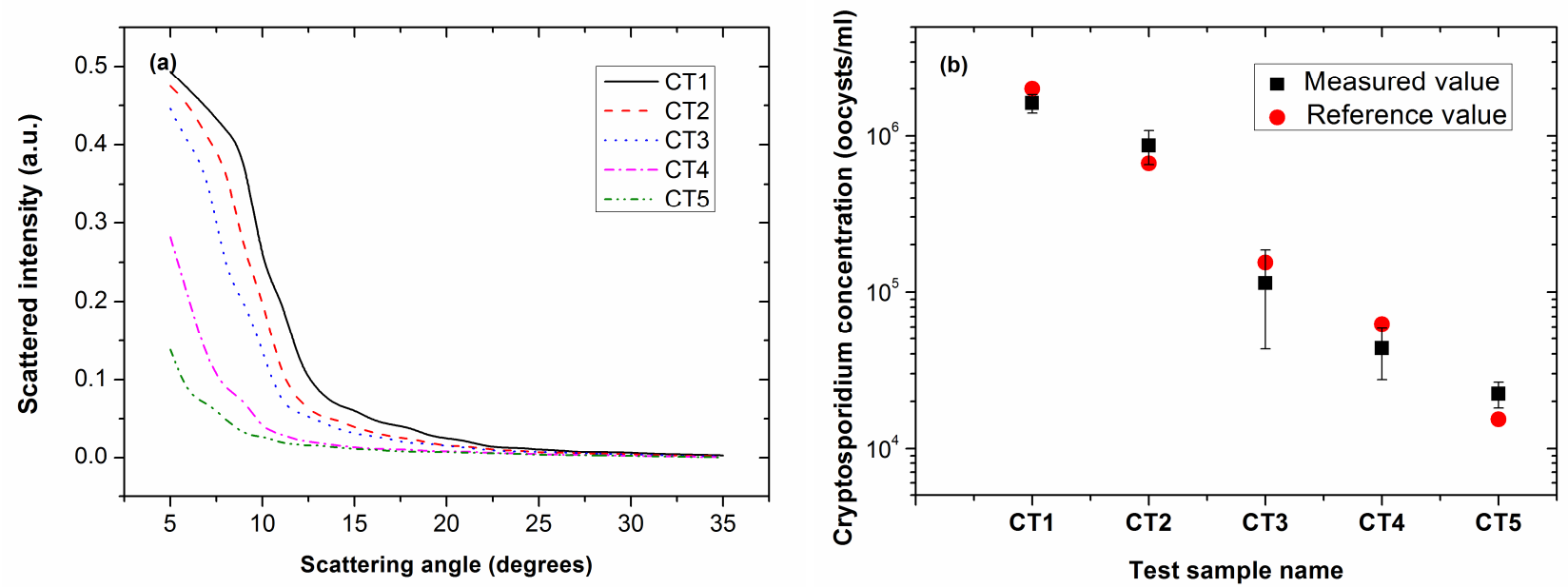

Figure 7 (a) Scattered light intensity of test samples in monodispersion. (b) Concentration measurement results for test samples of cryptosporidium in mono-dispersed solutions. 


\section{Mixed solution of cryptosporidium and latex beads}

In the mixed suspension, the experimental samples were prepared from a mixture of cryptosporidium oocysts suspension with concentration in the range of $10^{4}-10^{6}$ oocysts $/ \mathrm{ml}$ and polystyrenes sphere suspension (Supplied by Sigma-Aldrich, UK, UK) concentration in the range of $10^{4}-10^{6}$ particles $/ \mathrm{ml}$. The angle dependent scattered light intensity of the calibration samples are presented in figure 8 . The predictive model created from these calibration samples by the PLS-R method showed that the correlation between predicted and reference concentrations of the calibration samples were 0.970 for cryptosporidium and 0.986 for polystyrene spheres in mixed solution. These implied that the generated model can be used as a predictive model for the mixed of cryptosporidium oocysts and polystyrenes suspension. Test samples (CLBT1-6) for mixed solutions were prepared with different concentrations from the calibration samples. The scattered intensities from the test samples are presented in figure 9 . The concentrations of the cryptosporidium oocysts and polystyrene spheres were predicted from the scattered signals based on the predictive model by the PLS-R method. The predicted results are shown in figure 10.
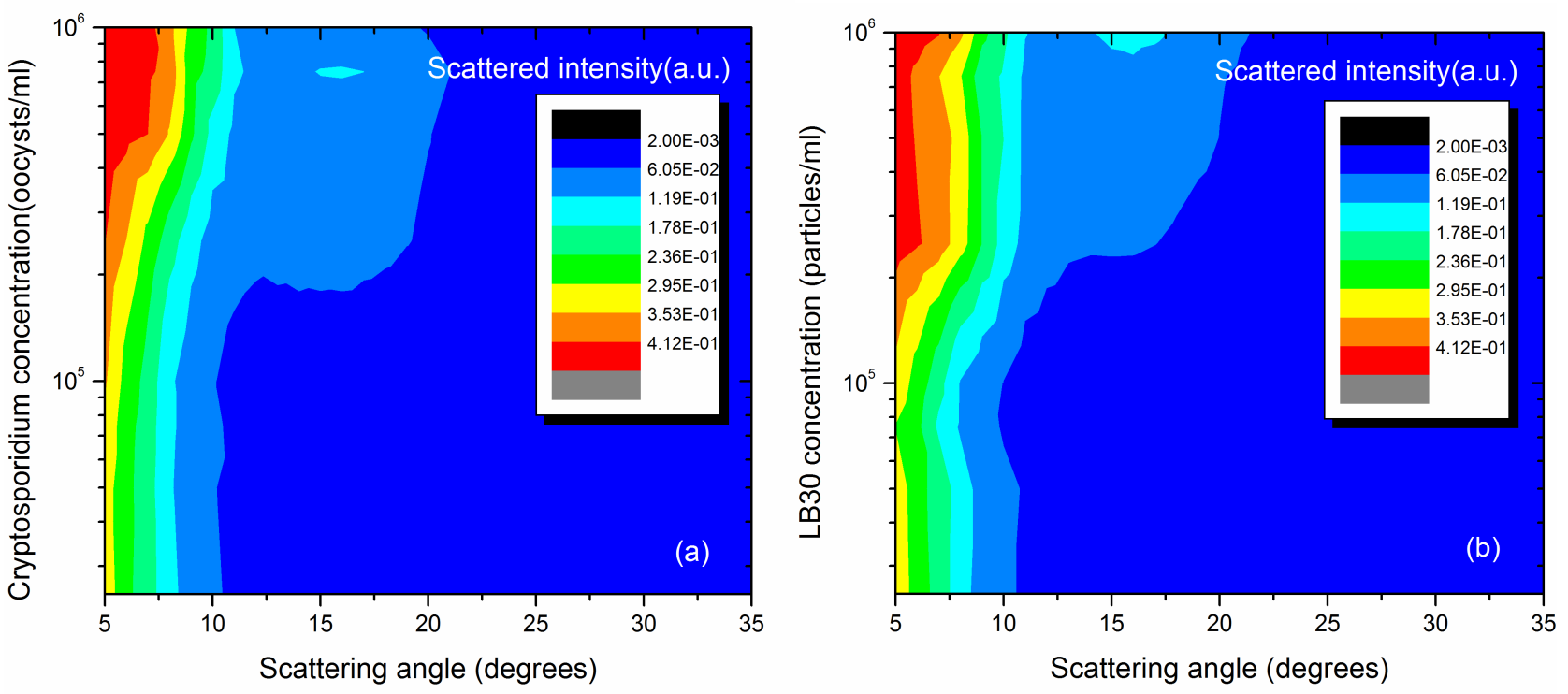

Figure 8 Angle dependent scattered intensities of calibration samples for mixed solutions of cryptosporidium and polystyrenes for (a) Cryptosporidium and (b) Polystyrene sphere.

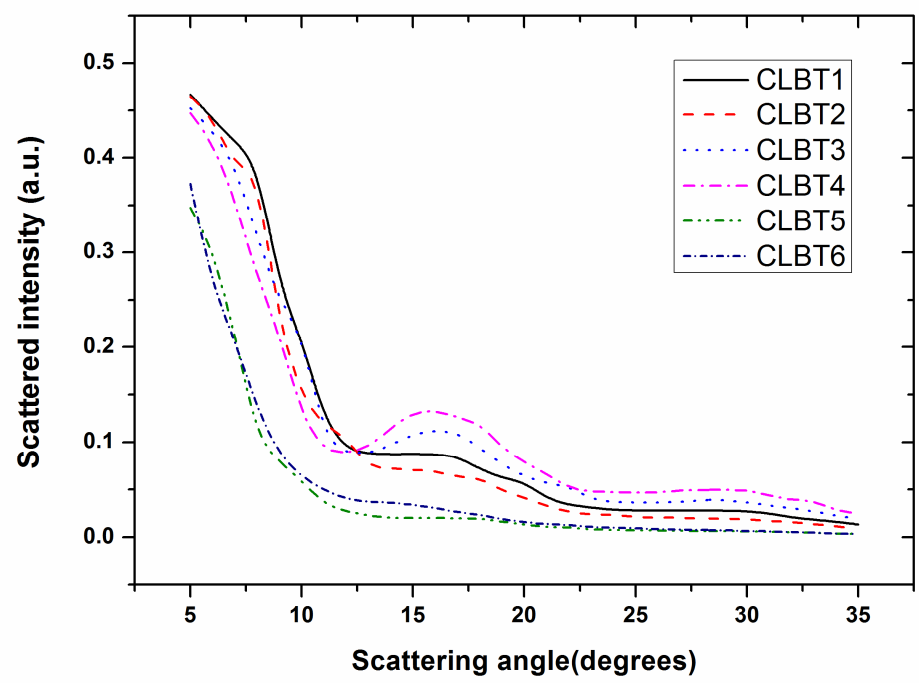

Figure 9 Angle dependent scattered intensities of test samples for mixed solutions of cryptosporidium and polystyrenes. 


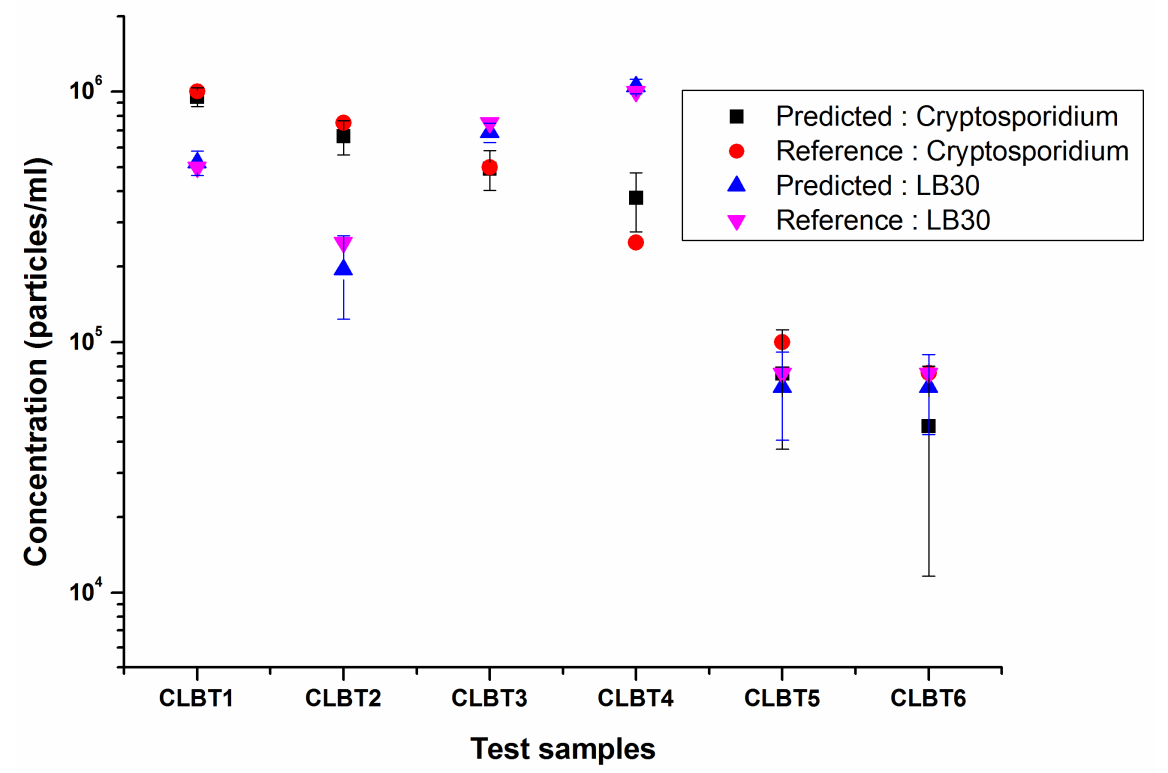

Figure 10 Concentration measurement results for test samples of mixed solutions of cryptosporidium and polystyrenes.

The concentration measurement results of cryptosporidiums in monodispersion (figure7) and cryptosporidiums and polystyrene spheres in mixed suspensions (figure10) show good correlation between the predicted and the reference values. The results obtained in the presented study clearly demonstrated that the proposed system can be successfully used to differentially measure particle concentrations in suspension. The present system could be further developed to online concentration measurement.

\subsection{Dual multi-angle light scattering for cryptosporidium detection}

It has been demonstrated that forward scatter and side scatter can be used to identify a number of cell subpopulation since forward scattering is proportional to the diameter of the cell and side scattering with perpendicular light scatter is also sensitive to small internal structure and to refractive index change ${ }^{26}$. In addition, the scattering is also a function of wavelength ${ }^{31}$. For this reason, the proposed dual wavelength multiple angle light scattering system was developed for particle classification based on scattering patterns. The presented system was applied to suspensions of polystyrenes latex spheres size $0.1 \mu \mathrm{m}$ (LB1), $0.3 \mu \mathrm{m}$ (LB3), $1.1 \mu \mathrm{m}$ (LB11) and $3.0 \mu \mathrm{m}$ (LB30), E.coli and cryptosporidium oocysts. The relative wavelength scattered intensities of red to violet light $(\mathrm{R} / \mathrm{V})$ from the particle in suspension were collected in multiple scattering angles from $5^{\circ}-65^{\circ}$. The relative scattered light from each particle were presented in figure 11 and comparison of relative scattered light from each sample is shown in figure 12. 

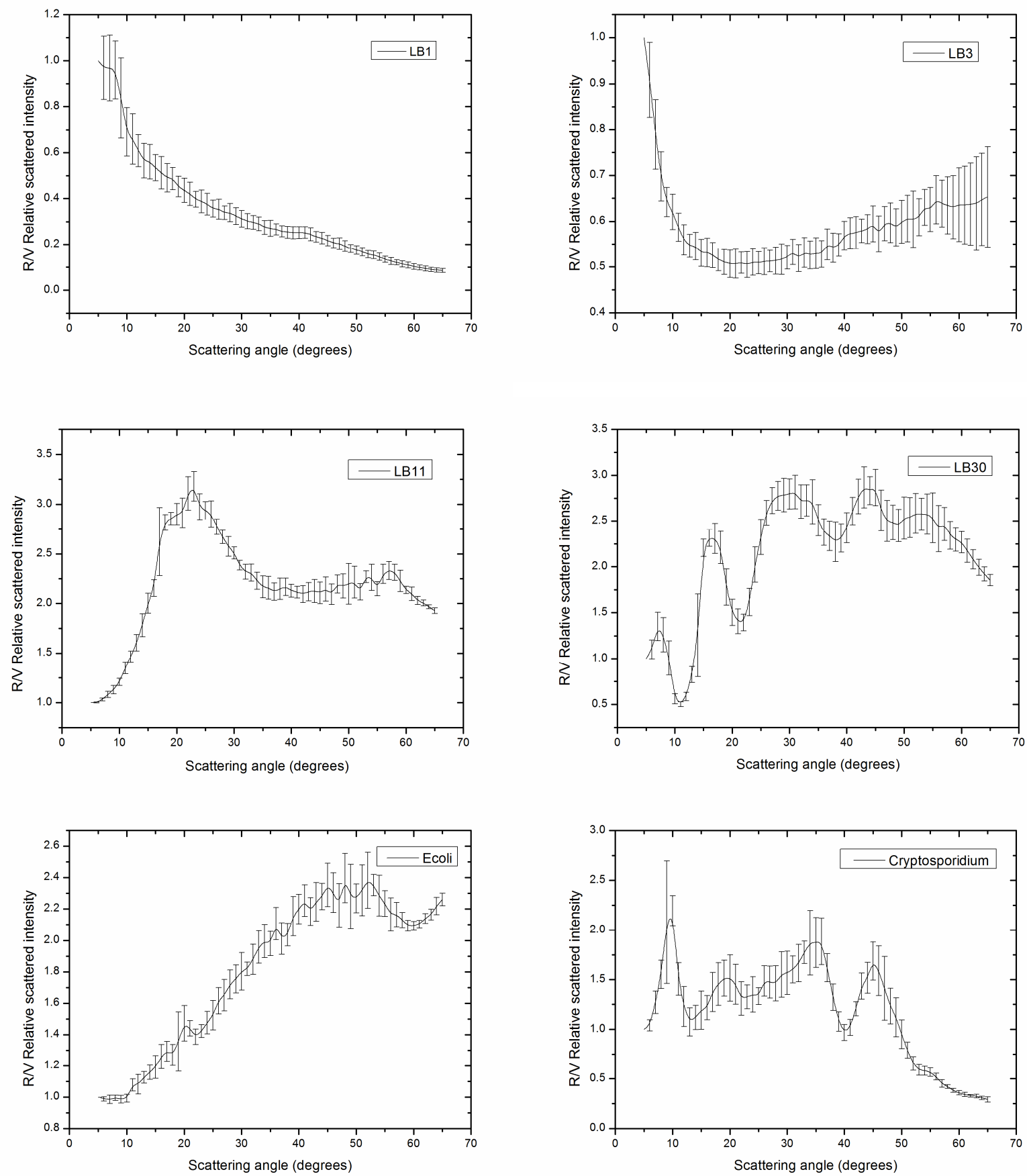

Figure 11 The relative wavelength scattered intensities of red to violet light from suspension of polystyrenes spheres, E.coli. and cryptosporidium oocysts. 


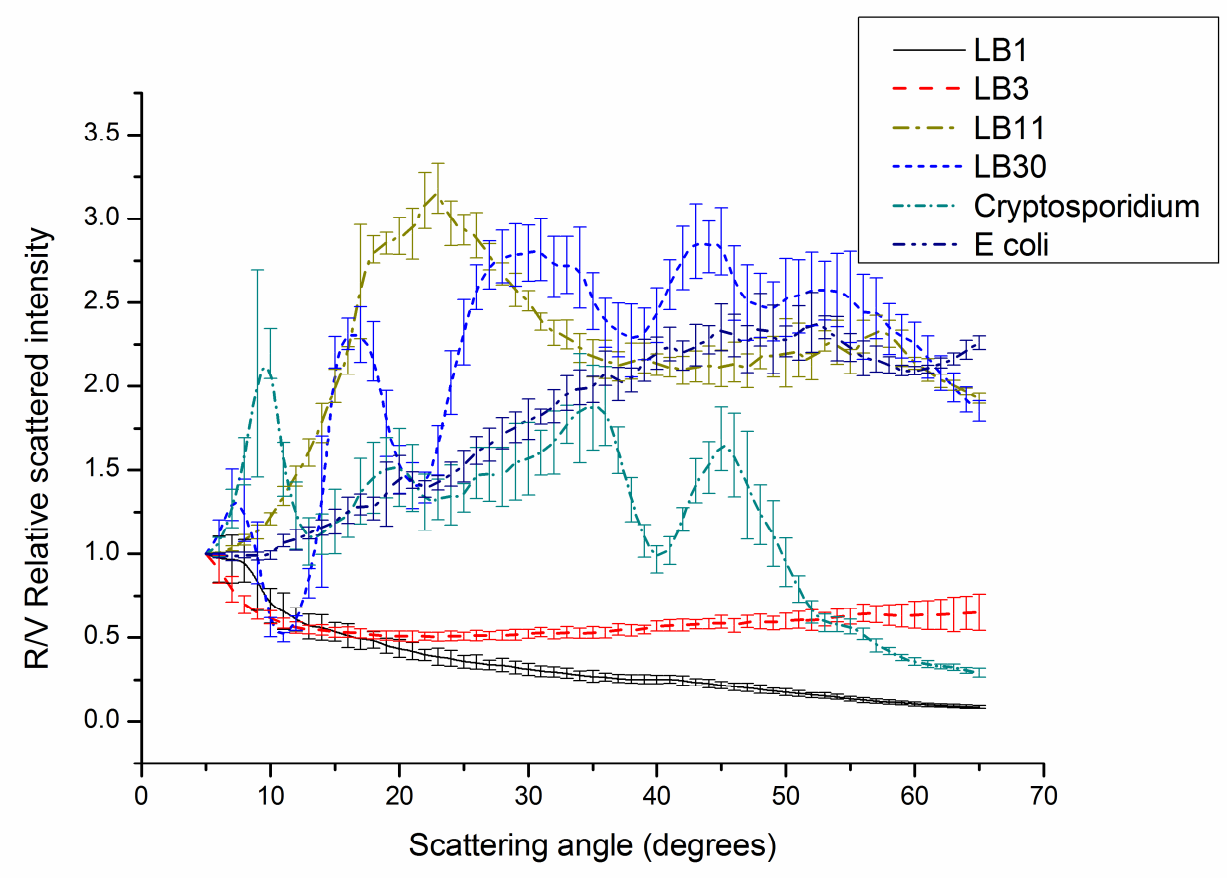

Figure12 Comparison relative wavelength scattered intensity of red to violet wavelength of polystyrenes, cryptosporidium oocysts and E.coli.

The results from figure11 and 12 show that the relative scattering pattern for each sample have significantly different patterns. The repeated measurements which are presented as error bar also show that the relative scattering pattern for each sample has a certain range of the pattern. These results have demonstrated that the proposed dual wavelength multiple angle light scattering system which has the advantage of scattering as a function of scattering angle, wavelength and polarization of the light can be further developed as a means of particle selection by combination to the pattern recognition technique.

\section{CONCLUSIONS}

A simple, dual wavelength multiple-angle light scattering system has been developed to initially identify cryptosporidium oocysts suspended in water. The proposed system was applied to classify particles by using relative wavelength scattering pattern technique. Testing with polystyrene spheres $0.1,0.3,1.1$ and $3.0 \mu \mathrm{m}$ size, E.coli and cryptosporidium oocysts suspended in water showed that the relative scattering pattern for each sample are significantly different from others. In addition, the proposed system with single wavelength operation was applied to identify cryptosporidium oocyst by the size and refractive index. The measured size correlated to the result measured by the SEM and the refractive index was consistent with the reference value. It was also used to differentially measure the concentrations of both cryptosporidium oocysts suspended in water and mixtures of cryptosporidium oocysts and polystyrenes latex beads in aqueous suspension. The measurement results showed good agreement with the control reference values.

The authors acknowledge financial support from the Thai Royal Government. 


\section{REFERENCES}

[1] Casemore D. P., Sands R. L. and Curry A. "Cryptosporidium species a new human pathogen”. J. Clin Pathol. 38, 1321-1336 (1985).

[2] Chappell C. 1., Okhuysen P. C., Sterling C. R., Wang C., Jakubowski W., and DuPont H. L., "Infectivity of Cryptosporidium parvum in healthy adults with pre-existing anti-C. parvum serum immunoglobulin G." Am J Trop Med Hyg 60(1), 157-164 (1999).

[3] Fayer R, Speer CA, Dubey JP. "The general biology of Cryptosporidium. In: Fayer R. (ed). Cryptosporidium and Cryptosporidiosis". CRC Press Boca Raton, Florida USA, 1-42(1997).

[4] Levine ND., "Taxonomy and review of the coccidian genus Cryptosporidium (Protozoa, Apicomplexa)", J. Proozool 31, 94-98 (1984).

[5] DWI, "Review of outbreaks of cryptosporidiosis in swimming pools", FWR, Allen House, The Listons, Marlow, Bucks., UK. (2000).

[6] Masago Y, Katayama H, Hashimoto A, Hirata T, Ohgaki S., "Assessment of risk of infection due to Cryptosporidium parvum in drinking water." Water Sci. Technol. 46(11/12), 319-324 (2002).

[7] Johnson, D.W., Pieniazek, N.J., Griffin, D.W., Misener, L. and Rose, J.B., "Development of a PCR protocol for sensitive detection of Cryptosporidium oocysts in water samples." Appl. and Environ. Microbiol. 61, 38493855(1995).

[8] Xiao, L., A. A. Lal, and J. Jiang., "Detection and differentiation of Cryptosporidium oocysts in water by PCRRFLP. Methods", Mol. Biol. 268, 163-176 (2004).

[9] Stinear, T., Matusan, A., Hines, K. and Sandery, M. "Detection of a single viable Cryptosporidium parvum oocyst in environmental water concentrates by reverse transcription-PCR." Appl. and Environ. Microbiol. 62, 3759-3763 (1996).

[10] Lindquist, H.D.A. "Probes for the specific detection of Cryptosporidium parvum." Water Research 31, 26682671 (1997).

[11] Slifco T.R., Friedman D., Rose J.B. and Jakubowski W., "An in vitro method foe detecting infectious Cryptosporidium oocysts with cell culture.” Appl. Environ. Microbiol.63, 3669-3675 (1997).

[12] Joan B. R., Lee K. L., Kelley R. R., and Charles P. G., "Evaluation of Immunofluorescence Techniques for Detection of Cryptosporidium Oocysts and Giardia Cysts from Environmental Samples", Appl. and Environ. Microbiol.55, 3189-3196 (1989).

[13] T. Chesnota, X. Marlyb, S. Chevalierb, and J. Schwartzbroda, "Optimised immunofluorescence procedure for enumeration of Cryptosporidium parvum oocyst suspensions," Water Research 36, 3283-3288 (2002).

[14] I. Weiner, M. Rust, T. D. Donnelly, "Particle size determination: An undergraduate lab in Mie scattering" Ame. J. Phys., 69 (2), (2001).

[15]B. Veihelmann, M. Konert, and W. J. van der Zande., "Size distribution of mineral aerosol: using lightscattering models in laser particle sizing", Appl. Opt. 45(23), 6022-6029 (2006).

[16] J. L. Castagner and I. J. Bigio., "Particle sizing with a fast polar nephelometer", Appl. Opt. 46(4), 527532(2007).

[17]Z. Ulanowski, R. S. Greenaway, P. H. Kaye, and I. K. Ludlow., "Laser diffractometer for single-particle scattering measurements", Meas. Sci. Technol. 13, 292-296 (2002).

[18] William D. Dicka; Paul J. Ziemannb; Peter H. McMurryc., "Multiangle Light-Scattering Measurements of Refractive Index of Submicron Atmospheric Particles" Aero. Sci. and Tech.41, 549-569 (2007).

[19] Shaughnessy, E. J.; Morton, J. B., "Laser light-scattering measurements of particle concentration in a turbulent jet" J. Flui. Mech.80, 129-148 (1977).

[20] Alfredo J. Armendariz , David Leith., "Concentration measurement and counting efficiency for the aerodynamic particle sizer 3320 " Aero. Sci.33, 133-148(2002).

[21] S. E. Harding, "Applications of light scattering in microbiology," Biotechnol. Appl. Biochem, 8 (6),489-509 (1986).

[22] B. V. Bronk, W. P. Van de Merwe, and M. Stanley, "In vivo measure of average bacterial cell size from a polarized light scattering function," Cytometry 13(2), 155-162 (1992).

[23] M. Bartholdi, G.C. Salzman, R.D. Hiebert and M. Kerker, "Differential Light-Scattering Photometer for Rapid Analysis of Single Particles in Flow", Appl. Opt.19(10), 1573-1581 (1980).

[24] A. Katz, A. Alimova, M. Xu, E. Rudolph, M. K. Shah, H. E. Savage, R. B. Rosen, S. A. McCormick, and R. R. Alfano, "Bacteria Size Determination by Elastic Light Scattering", IEEE J. Sel. Quant. Elec.9, (2003). 
[25] F. V. Ignatovich, D. Topham, and L. Novotny, "Optical Detection of Single nanoparticles and Viruses", IEEE J. sel. Quant. Elec.12, (2006).

[26] B. Rajwa, M. Venkatapathi, K. Ragheb, P. P. Banada, E. D. Hirleman, T. Lary, J. P. Robinson, "Automated Classification of Bacterial Particles in Flow by Multiangle Scatter Measurement and Support Vector Machine Classifier", Cytometry 73, 369-379 (2008).

[27] Kleinbaum, David G., D., Kupper L., Muller K.,and Nizam A., "Applied Regression Analysis and Multivariable Methods", Pacific Grove, Ca.: Brooks/Cole Publishing Company, (1998).

[28] D. Lehner, G. Kellner, H. Schnablegger and O. Glatter., "Static Light Scattering on Dense Colloidal Systems: New Instrumentation and Experimental Results", J. Coll. and Interf. Sci. 201(1), 34-47(1998).

[29] H.C. Van de Hulst, "Light Scattering by Small Particles", Wiley \& Sons, New York, (1957).

[30] J. Gregory, "Cryptosporidium in Water: Treatment and Monitoring Methods", Filtration \& Separation, 283-289 (1994).

[31]D. Chamberlin and R. Trutna, "Physics of Particle Size Spectrophotometry", Technical Note, Agilent Technologies, (2008). 